\title{
ROLE OF HYPERCORTISOLEMIA IN PROGRESSION OF THE COMORBID COURSE OF CORONARY ARTERY DISEASE, DIABETES MELLITUS TYPE 2 AND ANEMIA
}

\author{
Nataliia Pavliukovych ${ }^{1}$, Oleksandr Pavliukovych ${ }^{2}$, Oleksandr Buriak ${ }^{3}$, \\ Tamara Kopchuk $^{4}$, Volodymyr Vivsyannuk ${ }^{5}$
}

\begin{abstract}
:
INTRODUCTION: Hyperactivation of stress-limiting mechanisms of the organism is a unique physiological response of the human being to chronic hypoxia of different origins.

OBJECTIVES: Investigation of dynamics of cortisol synthesis changes in patients with coronary artery disease with comorbid diabetes mellitus type 2 and anemic syndrome in patients of older age groups.

METHODS: Blood cortisol level was measured in 40 old and senile coronary artery disease patients with comorbid diabetes mellitus type 2 and anemic syndrome of different degrees of severity. The control group consisted of 12 patients with coronary artery disease without comorbidities that were not significantly different through gender and age from the patients of the research groups. Possible effects of telmisartan on the degree of chronic hypoxia were also investigated.

RESULTS: In patients of old and senile age with coronary artery disease and comorbid anemic syndrome, same as in case of coronary artery disease and anemic syndrome on the background of diabetes mellitus type 2, activation of the cortisol synthesis is observed, likely in response to hypoxia as a stress factor in anemia. The degree of severity of the detected changes was different depending on the degree of anemia severity. There was no normalization of cortisol content in blood in any of the major experimental groups regardless the prescribed treatment with ACE inhibitors or telmisartan.

CONCLUSIONS: In patients with coronary artery disease, same as in the case of a comorbid course of coronary artery disease and diabetes mellitus type 2, an increase of cortisol secretion in response to anemic hypoxia was observed. As the degree of severity of the anemic syndrome progressed, gradual decreasing of cortisol blood level was detected. Reduction of the intensity of stress-limiting systems in the organism by telmisartan prescription may help to eliminate the adverse effects of hypercortisolemia on the progression of coronary artery disease, especially on the background of comorbid diabetes mellitus type 2 and anemia.
\end{abstract}

UDC Classification: 616.1, DOI: 10.12955/cbup.v7.1457

Keywords: coronary artery disease, diabetes mellitus, anemia, cortisol

\section{Introduction}

The human stress response has evolved to maintain homeostasis under conditions of real or perceived stress (Russell and Lightman, 2019). This objective is achieved through autoregulatory neural and hormonal systems in close association with central and peripheral clocks. The hypothalamic-pituitaryadrenal axis is a key regulatory pathway in the maintenance of these homeostatic processes. Cortisol, produced by the hypothalamus-pituitary-adrenal axis during a stress response, is still not included in the routine evaluation of cardiovascular risk and requires additional and definitive validation (Cozma et al., 2017).

In acute stress, hypersecretion of cortisol in the body is observed. There are many possible pathological reactions associated with this fact according to Kawada T. (2016): sympatho-adrenal activation with susceptibility to vasoconstriction, decreased heart rate variability, hyper-aggregation of thrombocytes, increased heart rate, arterial hypertension, increased C-reactive protein and proinflammatory cytokines levels, interleukin-1 and interleukin-6 secretion. Hammer et al. (2016) and Neary et al. (2013) suggested that increased levels of cortisol also contribute to the development and progression of heart failure and atherosclerotic processes. Through these mechanisms stress can provoke cardiovascular diseases.

\footnotetext{
${ }^{1}$ Higher education institution in Ukraine «Bukovinian State Medical University», Chernivtsi, Ukraine, natasha.pavlyukovich@gmail.com

${ }^{2}$ Higher education institution in Ukraine «Bukovinian State Medical University», Chernivtsi, Ukraine, olexandr.pavlyukovich@gmail.com

${ }^{3}$ Higher education institution in Ukraine «Bukovinian State Medical University», Chernivtsi, Ukraine, buriakoleksandr1983@gmail.com

${ }^{4}$ Higher education institution in Ukraine «Bukovinian State Medical University», Chernivtsi, Ukraine, toma.kopchuk@gmail.com

${ }^{5}$ Higher education institution in Ukraine «Bukovinian State Medical University», Chernivtsi, Ukraine, dr.vivsyannuk@yandex.ua
} 


\section{Aim}

Investigation of changes of the cortisol secretion in patients with coronary artery disease with comorbid diabetes mellitus type 2 and anemic syndrome in patients of older and senile age and determining possible interconnection between revealed changes and anemic hypoxia severity.

\section{Material and Methods of Investigation}

Due to the fact that synthesis of certain glucocorticoid in stress conditions is species-specific, and human beings are characterized by cortisol secretion, namely by the level of this hormone in the blood serum we have estimated glucocorticoid function of the adrenal cortex.

The level of blood cortisol was measured in 40 old and senile coronary artery disease (CAD) patients with comorbid diabetes mellitus type 2 (DM) and anemic syndrome (AS) of different degrees of severity. Average age of the investigated patients was $67 \pm 4.5$ years. The control group consisted of 12 patients with CAD without comorbidities that were not significantly different through gender and age from the patients of the research groups. All patients were treated for their main and comorbid diseases (aspirin, beta-blocker, statin, ACE inhibitor), additionally telmisartan was prescribed. Therefore, patients with $\mathrm{CAD}, \mathrm{DM}$ and anemia additionally were randomized into groups depending on the prescribed treatment: A - 20 patients who received only basic therapy and B -20 patients whom in the scheme of the basic treatment replacement of ACE inhibitor by angiotensin receptor blockers II telmisartan was performed, the last was prescribed in a dose $40 \mathrm{mg}$ daily after meals. Duration of in-hospital treatment was 21-24 days, additionally telmisartan was prescribed for outhospital treatment over a period of 4-6 months.

Investigation of cortisol levels in blood plasma was performed by immunoassay analysis on the RT2100C immunoassay analyzer (Rayto Electronics Inc., China). A set of reagents Kortizol-IFA (OOO Hema-Medica, Russia) was used with a range of normal values from 150 to $650 \mathrm{nmol} / \mathrm{l}$.

\section{Results of the Investigation}

Cortisol content in blood serum of patients of the control group was $390.8 \pm 52.67 \mathrm{nmol} / \mathrm{l}$, which was within the physiological norm (Figure 1).

Figure 1: Cortisol level in serum of patients with coronary artery disease, diabetes mellitus type 2
and anemic syndrome
Note: * difference is valid against patients of control group ( $p<0,05)$; \#-difference is valid against patients
with CAD and DM $(p<0.05)$
Source: Author

We did not detect significant changes in cortisol blood level due to the comorbid course of CAD and DM: cortisol content in the blood serum was $476.7 \pm 39.11 \mathrm{nmol} / \mathrm{l}$ ( $\mathrm{p}>0.05$ compared with the control group), which also was within normal values. In patients with CAD and comorbid anemia of different degrees of severity, cortisol content was 2.02 times higher $(\mathrm{p}<0.05)$ and was $966.2 \pm 66.51 \mathrm{nmol} / \mathrm{l}$. In case of complication of CAD by DM and AS cortisol level in blood was slightly lower $(897.4 \pm 38.43 \mathrm{nmol} / \mathrm{l})$ and significantly different from the similar value in the control group $(\mathrm{p}<0.05)$ and patients with CAD and DM without anemia $(\mathrm{p}<0.05)$. 
Thus, as in patients with CAD and comorbid AS, same as in case of CAD and AS on the background of DM, activation of the glucocorticoid function of the adrenal cortex and significant hypercortisolemia are observed, likely in response to hypoxia as a stress factor in anemia.

Taking into consideration higher incidence of mild anemia compared with moderate and severe degree among patients with CAD and DM type 2, it can be assumed that the effect and course of the disease affect the degree of hypercortisolemia. This made us compare the values of the cortisol content in serum in patients of the main groups depending on the severity of the comorbid AS.

As seen in Figure 2, in patients with CAD and mild anemia compared with patients of the control group, significant increase in cortisol blood content was observed. Obviously, anemia aggravates hypoxia, which occurs in CAD patients due to decreased cardiac output and insufficient perfusion of organs and tissues, which results in compensatory activation of cortisol secretion. With progression of anemia severity, we observed the tendency towards the depletion of the glucocorticoid function of the adrenal cortex with a decrease in the content of serum cortisol, which, however, was not statistically significant ( $\mathrm{p}>0.05)$. For CAD and anemia of moderate severity cortisol level was $883.2 \pm 87.78$, which was $10 \%$ less than in patients with anemia of a mild degree.

Figure 2: Cortisol level in serum of patients with coronary artery disease and diabetes mellitus type 2 depending on the anemic syndrome severity

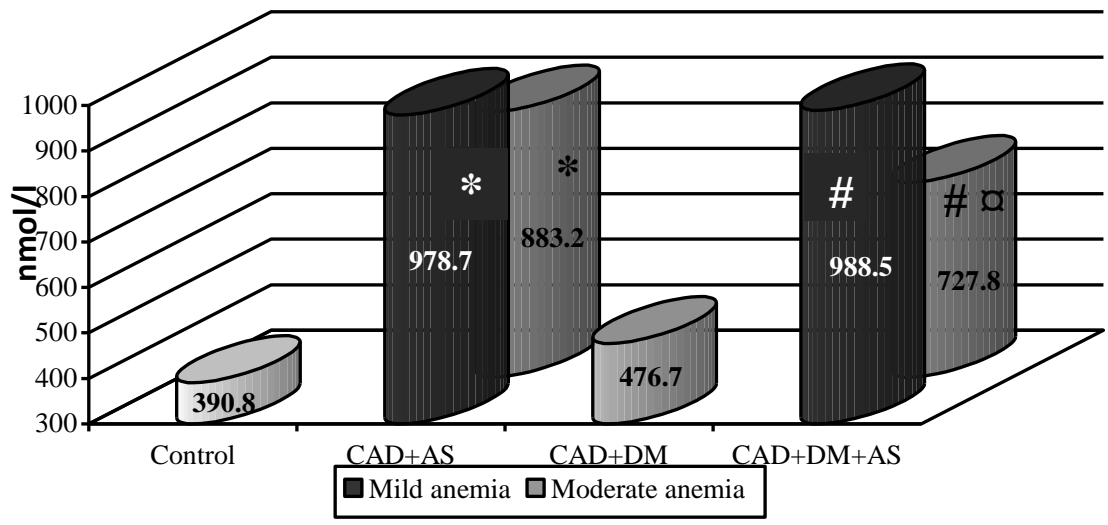

Note: * difference is valid against patients of control group $(p<0,05) ; \#$ - difference is valid against patients with $C A D$ and DM ( $p<0.05) ; \quad \alpha$ - difference is valid against patients with CAD, DM and mild anemia $(p<0.05)$

Source: Author

Similar tendency of the changes of cortisol blood level due to the degree of anemic hypoxia was observed in the case of the comorbid course of CAD, DM and anemia. In patients with CAD and DM comorbid to mild anemia there was a statistically significant increase of the cortisol content of blood serum of 2.07 times compared with patients with CAD and DM ( $\mathrm{p}<0.05)$, which may be considered as activation of the stress-limiting possibilities of the organism in response to hypoxia. Along with progression of the comorbid anemia degree of severity, there was a progressive depletion of the glucocorticoid function of adrenal glands, accompanied by a statistically significant decrease in cortisol blood content by $26 \%$ compared with the same figure in patients with CAD, DM and mild AS $(\mathrm{p}<0.05)$, the values of which, however, did not reach the corresponding value in the group of patients with CAD and DM.

As a result of the conducted treatment, patients in both experimental groups were characterized by a certain dynamics of changes in the cortisol blood level, which was manifested by a marked tendency towards the reduction of the hypercortisolemia in the blood serum of patients with CAD, DM type 2 and comorbid anemia (Figure 3). The degree of severity of the detected changes was different depending on the type of treatment.

In patients of group A, treated by the standard scheme of treatment (aspirin, beta-blocker, statin, ACE inhibitor), cortisolemia decreased by $12 \%$ compared with its corresponding values before treatment (771.9 \pm 93.10 and $874.5 \pm 41.12 \mathrm{nmol} / \mathrm{l}$, respectively), which, however, was not statistically significant 
( $>>0.05)$ and 1.97 times higher than that in the control group $(p<0.05)$. The average level of cortisol in patients of group B (where ACE inhibitors were replaced by telmisartan because of low adherence of the patients to prolonged treatment with ACE inhibitor) was $663.7 \pm 96.69 \mathrm{nmol} / \mathrm{l}$, statistically significantly differing by $27 \%$ for its corresponding value before treatment $(\mathrm{p}<0.05)$. There was no normalization of cortisol content in blood in any of the major experimental groups.

Figure 3: Cortisol level in serum of patients with coronary artery disease, diabetes mellitus type 2 and anemia depending on the prescribed treatment

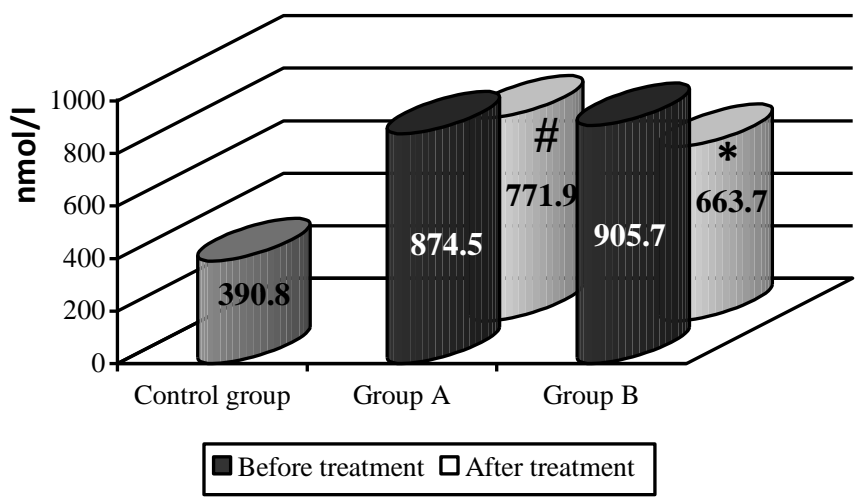

Note: * - difference is valid against patients before and after treatment $(p<0.05)$; \# - difference is valid against patients of control group $(p<0.05)$

Source: Author

\section{Conclusion}

Thus, in patients with CAD and anemia, same as in the case of a comorbid course of CAD, AS and DM, an increase of cortisol secretion in response to anemic hypoxia was observed. As the degree of severity of the comorbid AS progressed, gradual significant decreasing of hypercortisolemia level was detected. Therefore, chronic hypoxia in patients with comorbid course of CAD, DM and anemia, particularly of a mild degree of severity, may contribute to constant hypercortisolemia, progression of $\mathrm{CAD}$ and increasing of general cardiovascular risk. Progression of comorbid anemia severity in such patients may partially play a protective role as it is characterized by the decreasing of the hypercortisolemia degree. Reducing the intensity of stress-limiting systems in the organism may help to eliminate the adverse effects of hypercortisolemia on the progression of coronary artery disease, especially on the background of comorbid diabetes mellitus type 2 and anemia. Prescription of telmisartan in such patients may have some benefits over ACE inhibitors taking into consideration its positive effects on the decreasing of blood cortisol levels.

\section{References}

Cozma S., Dima-Cozma L.C., Ghiciuc C.M., Pasquali V. (2017) Salivary cortisol and $\alpha$-amylase: subclinical indicators of stress as cardiometabolic risk. Brazilian journal of medical and biological research. 50 (2). e5577. https://dx.doi.org/10.1590/1414-431X20165577.

Hammer F., Deutschbein T., Marx A., Güder G. (2016) High evening salivary cortisol is an independent predictor of increased mortality risk in patients with systolic heart failure. International journal of cardiology. 203. 69-73. https://dx.doi.org/10.1016/j.ijcard.2015.10.084.

Kawada T. (2016) Survival risk of salivary cortisol and serum N-terminal pro-hormone B-type natriuretic peptide in patients with systolic heart failure. International journal of cardiology. - 2016. 222. 1054.

https://dx.doi.org/10.1016/j.ijcard.2016.01.192.

Neary N.M., Booker O.J., Abel B.S., Matta J.R. et al. Hypercortisolism is associated with increased coronary arterial atherosclerosis: analysis of noninvasive coronary angiography using multidetector computerized tomography (2013). J. Clin. Endocrinol. Metab. 98(5):2045-52. https://dx.doi.org/10.1210/jc.2012-3754.

Russell G., Lightman S. (2019) The human stress response. Nat Rev Endocrinol. 15(9):525-534.

https://dx.doi.org/10.1038/s41574-019-0228-0. 